\title{
Modification of Thick-film Conductors Used in IP Technology for Reduction of Warpage during Co-firing of LTCC (Low Temperature Co-fired Ceramic) Modules
}

\author{
Hansu Birol, Thomas Maeder and Peter Ryser \\ Ecole Polytechnique Fédérale de Lausanne (EPFL), Laboratoire de Production Microtechnique \\ (LPM), CH-1015, Lausanne, Switzerland
}

Keywords: LTCC, differential shrinkage, warpage, shrinkage matching, mixing.

\begin{abstract}
LTCC technology offers low temperature firing $\left(<900{ }^{\circ} \mathrm{C}\right)$ of a materials system, which is based on LTCC sheets/tapes and (ideally) compatible thick-film components. Screen-printed materials on LTCC tapes, such as conductor, resistor, inductor thick-films are co-fired (simultaneously fired), providing a highly-functional package. This comes along with additional benefits such as ease of LTCC tape structuring, fabrication of hermetic and complex 3-D structures, etc. The major difficulty encountered arises from the differential shrinkage rate of LTCC tape and thick-film components, which has to be avoided for fabrication of warpage-free, flat surfaces that is vital for membranes, beams, etc. Therefore the goal of this study is the reduction of deformation, by matching the shrinkage rate of conductor with that of LTCC, which is achieved by mixing the commercially-available paste with selected additives.
\end{abstract}

\section{Introduction}

Increasing demands for downscaling and smart packaging of electronic components in microengineering have recently led to the use of LTCC technology as an alternative solution for various applications. Sensors, micro-fluidics, MEMS are some examples, which actually differ than the traditional high-frequency applications which have received the major attention for more than 20 years [1-5]. The versatility of applications is ascribed to the ease of 3-D structuring of tapes, thermal and chemical stability and hermeticity of the fired structures, where multiple functions (mechanical, electrical and fluidic) can be integrated.

One of the main functionality aspects of the technology is co-firing passive electronic components on LTCC sheets [6]. This is realized by screen-printing thick-film conductors, resistors, capacitors, etc on/in (surface/buried) LTCC, which is followed by firing the overall module in oxidizing atmosphere. Although this is straightforward at first look, co-firing introduces difficulties, which arises from incompatibility of different materials [7-10]. Among those, warpage, which occurs due to differential shrinkage between the tape and the printed thick-films, is the most widely-observed. The films start densification at relatively lower temperatures than that of LTCC sheet, deforming the tape prior to densification [11]. This issue is very critical in devices carrying thin LTCC structures such as membranes and beams, where this effect is pronounced and the performance of the device depends on achieving a precise, defect-free geometry.

Therefore, the objective of this study is to match the shrinkage rate of thick-film conductors with LTCC, which we have demonstrated to be a promising attempt to reduce the deformation without altering the electrical properties drastically. The method of the study is based on mixing commercial thick-film pastes, with selected additives at varying concentrations. Fabrication of warpage-free millinewton force sensor, which is the origin of our motivation to make this study, experimental details and results are explained in details. Dilatometer anaylsis, SEM (scanning electron microscopy), EDXS (electron dispersive X-ray) analysis will be basic tools for the study.

\section{Motivation and Experimental}

The starting point / necessity of this work originates from a previous project, in which we had aimed 
replacing the alumina-based beam of the force sensor with LTCC [11]. Although it is not the objective of this paper, some of the motives for such a replacement can be listed as: lower elastic modulus of LTCC providing higher strain, thinner substrates and design flexibility, all of which contribute to improved signal [11].

Both substrates serve as different platforms for integration of thick-film components during processing. In the alumina-based version, thick-film components (conductor and piezoresistor) are screen-printed and fired on rigid, dense alumina substrates, whereas in the LTCC-based version, the components are co-fired with raw, unfired LTCC sheet. The major problem occurs at this point, since each material (Table 1, list of materials used) has a characteristic densification behavior. The dilatometer analysis shows that the conductors start shrinking $150-240{ }^{\circ} \mathrm{C}$ prior to LTCC tape, (fig. 1.a) ending up with an increased overall shrinkage. This leads to deformation of the LTCC tape, which is yet not dense at such low temperatures (fig. 1.b). The mismatch in shrinkage rates is a serious problem as it deforms the beam of the force sensor, resulting in poor/no performance.

Table 1. Materials Used for Fabrication of Force Sensor

\begin{tabular}{l|l|l}
\hline \multicolumn{1}{c|}{ Substrates } & \multicolumn{2}{|c}{ Thick-film components } \\
\hline DuPont LTCC (95 I series) & DuPont 9473 (Ag/Pd) & DuPont 2041 (piezoresistor) \\
Kyocera A-476 $\mathrm{Al}_{2} \mathrm{O}_{3}(96 \%)$ & ESL 9562 (Ag/Pd/Pt) & \\
\hline
\end{tabular}
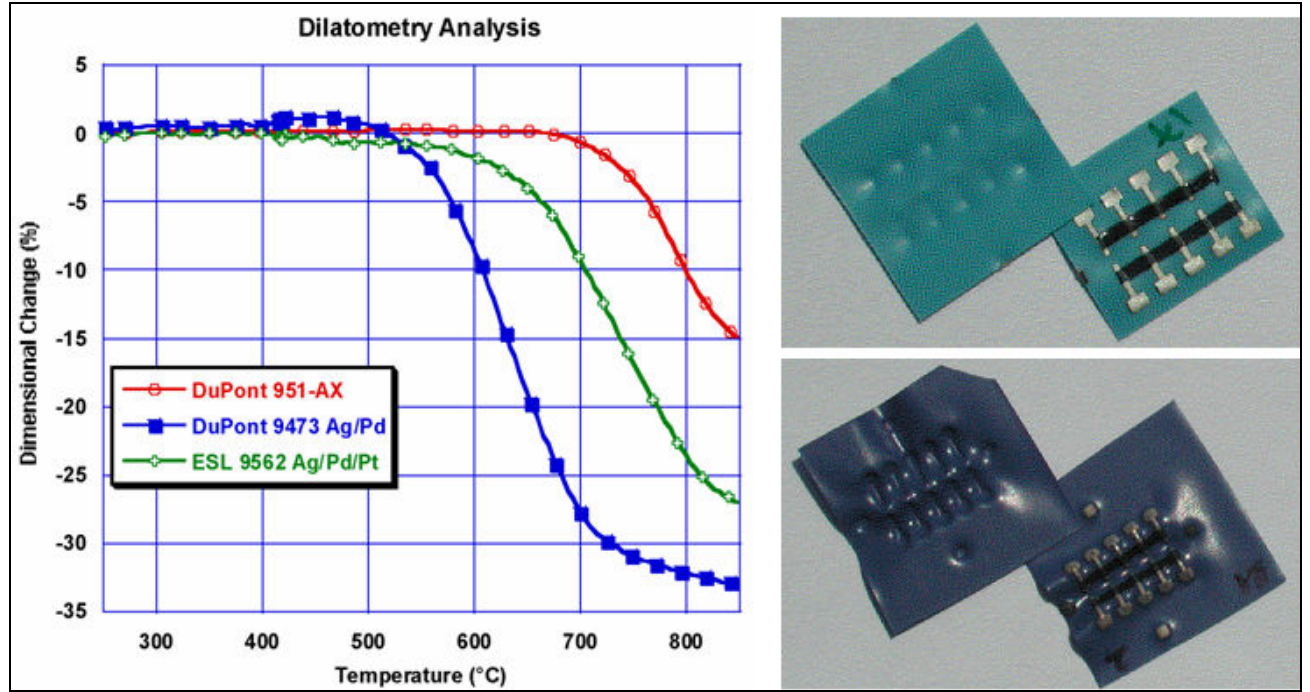

Fig. 1.a. Dilatometer analysis made on pellets of commercial products, b. Deformation of LTCC due to differential shrinkage with the thick-film components.

Table 2. Materials used for modification of pastes

\begin{tabular}{|c|c|c|c|}
\hline \multirow[b]{2}{*}{ Conductor } & \multirow[b]{2}{*}{ Specification } & \multicolumn{2}{|c|}{ Additive (w eight \%) } \\
\hline & & $\begin{array}{c}\mathrm{SiO}_{2} \\
\text { Sihelco, Sikron B600 }\end{array}$ & $\begin{array}{l}\text { LTCC } \\
\text { powder }\end{array}$ \\
\hline \multirow{2}{*}{ DuPont 9473} & \multirow{2}{*}{$\mathrm{Ag} / \mathrm{Pd} \sim 3 / 1, \mathrm{Zn}-\mathrm{Bi}-\mathrm{Si}$ based frit } & 10 & 10 \\
\hline & & 20 & 20 \\
\hline \multirow{2}{*}{ ESL 9562} & \multirow{2}{*}{$\mathrm{Ag} / \mathrm{Pd} / \mathrm{Pt} \sim 16 / 1 / 1$, fritless } & 10 & 10 \\
\hline & & 20 & 20 \\
\hline
\end{tabular}

Therefore, the commercial conductors have been modified in order to reduce the deformation on the beams, by matching their shrinkage behavior to that of LTCC. Two types of additives are mixed with the conductors at different ratios: silicate $\left(\mathrm{SiO}_{2}\right)$ and LTCC (table 2). The former compound is a network promoter [12] and known to increase the glass transition temperature, $\mathrm{T}_{\mathrm{g}}$, whereas the latter selection is a practical approach to match the shrinkage behavior of conductor to that of the LTCC tape. LTCC powder, which is blended with the commercial paste, is obtained by a series of processes, 
which can be summarized as: separation of the organic vehicle in the tape using acetone in a crucible whilst applying ultrasound, heating the mixture gradually up to $250^{\circ} \mathrm{C}$ and grinding the dried mixture.

The mixed conductor paste and the modifier (powder) are homogenized on a thee-roll mill. The resulting paste is screen-printed on test patterns and co-fired at $875^{\circ} \mathrm{C}$, in air. The pastes, yielding the minimum deformation are selected for force sensor application.

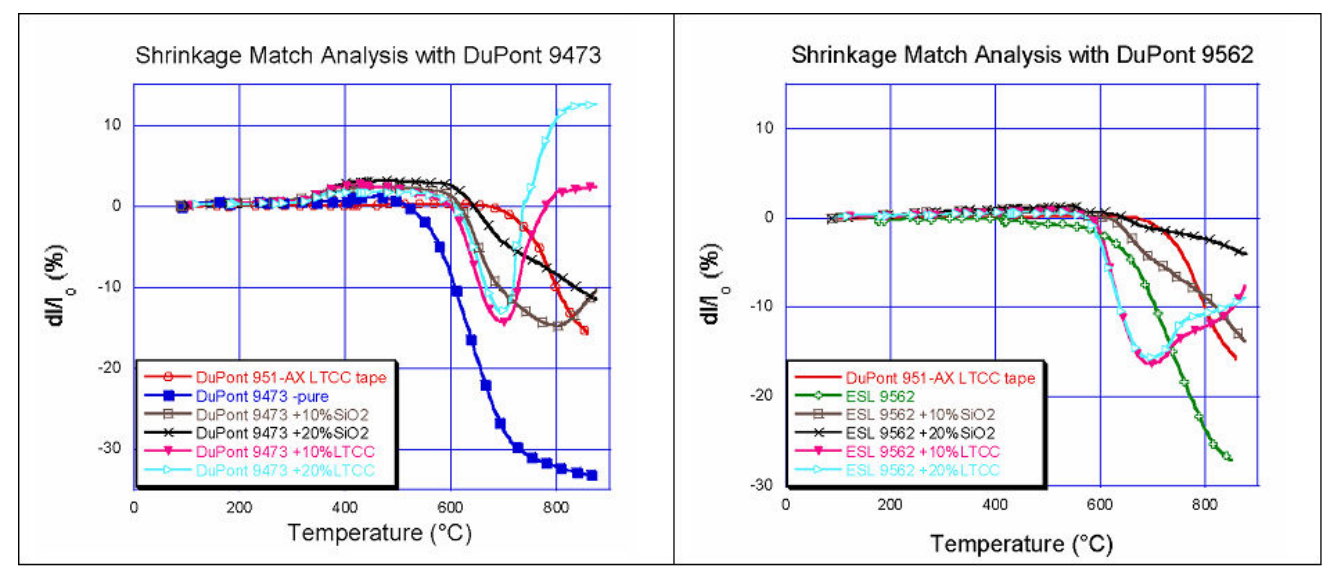

Fig. 2.a. Dilatometer analysis made on pellets of LTCC, pure and modified conductors

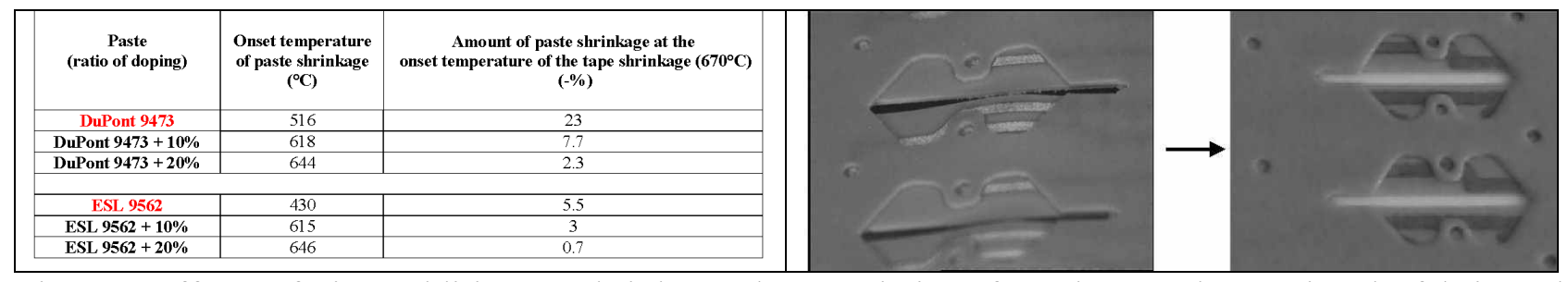

Fig. 3.a. Effects of $\mathrm{SiO}_{2}$-addition on shrinkage characteristics of conductors, b. Previously-fabricated beam (left) becoming flat and warpage-free after modification $\left(20 \% \mathrm{SiO}_{2}\right.$ by weight).

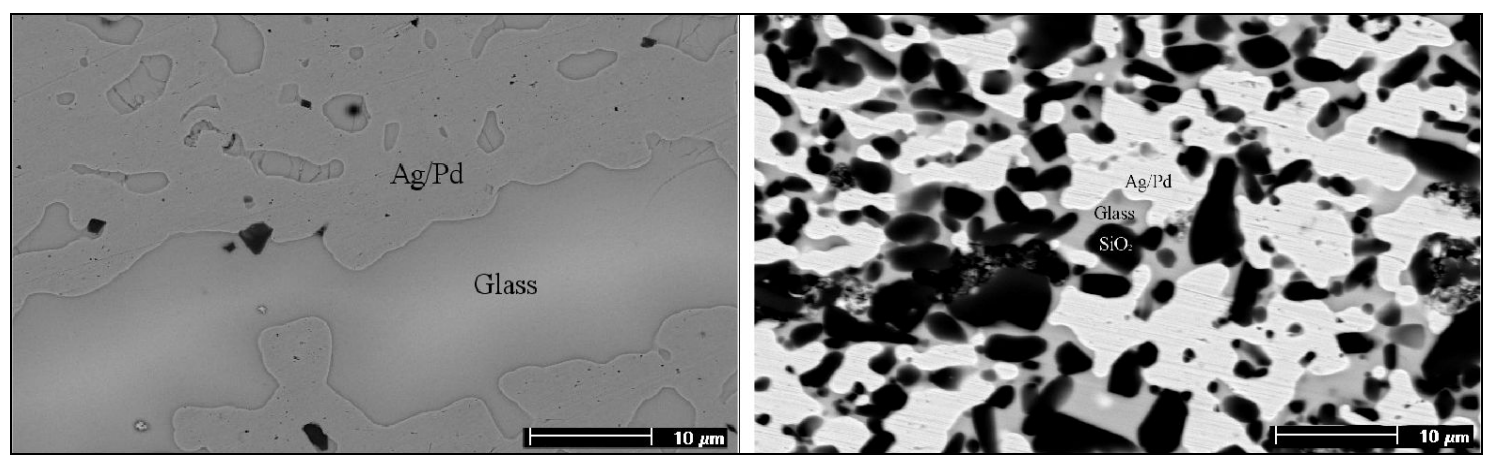

Fig. 4. Pellet of the pure (left) and modified conductor paste, DuPont 9473.

\section{Results and Discussions}

The results of the dilatometer analysis carried on pellets of LTCC tape, pure and modified pastes are shown in fig. 2. The effects of $\mathrm{SiO}_{2}$-addition on the onset temperature of shrinkage and the total shrinkage value of the conductors are separately shown in fig. 3.a. These results are also confirmed with LTCC beams, which are co-fired with modified, Ag/Pd-based DuPont conductor paste (9473), ending up with reduced deformation (fig. 3.b).

It is seen from the results that modification by addition of $\mathrm{SiO}_{2}$ particles changes the densification mechanism and reduces the deformation on the beams effectively. One can see the micro-structure of pellets prepared from pure and modified DuPont 9473 paste in fig. 4 . The pure pellet is composed of two main phases: the $\mathrm{Ag} / \mathrm{Pd}$ matrix and the glass phase (Bi-Zn) that is observed as rounded (particles) 
regions, typical of liquid phase sintering. The modified pellet, on the other hand, has both of these phases and $\mathrm{SiO}_{2}$, which is observed as black areas dispersed over glass regions. According to our preliminary analysis, we ascribe the change of densification mechanism to these particles, which hinder the mobility of the glass phase over $\mathrm{T}_{\mathrm{g}}$.

Mixing the pastes with LTCC powder follows a different trend than that of $\mathrm{SiO}_{2}$. Although the overall shrinkage is reduced, expansion around $700{ }^{\circ} \mathrm{C}$ (fig. 2.) leads to limited reduction of deformation of the fabricated beams.

\section{Conclusions}

This work, from an application point of view, summarizes the efforts to reduce warpage that occurs on the cantilever beam of the force sensor fabricated. The formation of this defect is ascribed to the differential shrinkage between the LTCC tape and the thick-film components and it is effectively reduced by modifying the commercial conductors. It is shown that addition of $\mathrm{SiO}_{2}$ particles remarkably shifts the onset of shrinkage temperature of the conductor closer to that of LTCC tape, by which the shrinkage rates are matched. Moreover this modification also reduces the overall shrinkage of the conductor considerably. Although it is a preliminary approach to explain the exact mechanism of this improvement, silicate additives are thought to hinder the mobility of the glass phase over, $\mathrm{T}_{\mathrm{g}}$ as it can be seen from SEM images and EDXS analysis.

\section{References}

[1] S. Annas: IEEE Elec. Comp. Tech. Conf. (2003), pp. 1691-1693.

[2] T. Thelemann, H. Thust and M. Hintz: European Microelectronics Packaging and Interconnection Symposium (2002), pp. 187-191.

[3] R. Kulka, M. Mittweger, P. Uhlig, C. Günther: IMST GmbH, http://www.ltcc.de (2001).

[4] M.G-Rubio, L.M.S-Laguna, P.E-Vallejos and J.J.S-Aviles: Sensor. Actuat. A-Phys. Vol. 89, (2001), pp. 222-241.

[5] H. Birol, T. Maeder, C. Jacq, G. Corradini, Y. Fournier, I. Saglini, S. Straessler and P. Ryser: Proc. 15th EMPC, Brugge-Belgium, (2005), 243-247.

[6] R.L.Brown, A.A.Shapiro, P.W. Polinski: Int. J. Micro. Elec. Pack. Vol. 16 (1993), pp. 328-338.

[7] C.J. Ting, C.S. His and H.Y. Lu: J. Amer. Ceram. Soc., Vol. 83/12 (2000), pp. 2945-2953.

[8] H. Birol, T. Maeder, C. Jacq and P. Ryser: J. Eur. Ceram. Soc. Vol. 25/12 (2005), pp. 2065-2069.

[9] H. Birol, T. Maeder, C. Jacq and P. Ryser: in publication at J. Eur. Ceram. Soc.

[10]P.Yang, M.A.Rodriguez, P.Kotula, B.K.Miera and D.Dimos: J. Appl. Phys. Vol. 89/7 (2001), pp. 4175-4182.

[11]H. Birol, T. Maeder, M. Boers, C. Jacq, G. Corradini and P. Ryser: Proc. Conference on Ph. D. Research in Micro-electronics and Electronics (PRIME 2005), Lausanne-Switzerland, Vol. 2 (2005), pp. 343-346.

[12]R.C. Sutterling, G.O. Dayton and J.V.Biggers: IEEE Trans. Comp. Pack. Manuf. Tech. Vol. 18 /Part B (1995), pp. 346-351. 